\title{
Research on Rolling Bearing Fault Diagnosis Using Improved Majorization-Minimization-Based Total Variation and Empirical Wavelet Transform
}

\author{
Yangli Ou, Shuilong He (D), Chaofan Hu, Jiading Bao, and Wenjie Li \\ School of Mechanical \& Electrical Engineering, Guilin University of Electronic Technology, Guilin, Guangxi 541004, China \\ Correspondence should be addressed to Shuilong He; xiaofeilonghe@163.com
}

Received 13 March 2020; Revised 25 April 2020; Accepted 2 May 2020; Published 15 May 2020

Academic Editor: Yongteng Zhong

Copyright () 2020 Yangli Ou et al. This is an open access article distributed under the Creative Commons Attribution License, which permits unrestricted use, distribution, and reproduction in any medium, provided the original work is properly cited.

\begin{abstract}
Bearings are among the most widely used core components in mechanical equipment. Their failure creates the potential for serious accidents and economic losses. Vibration signature analyses are the most common approach to assess the viability of bearings due to its ease of measurement and high correlation with structural dynamics. However, the collected vibration signals of rolling bearings are usually nonstationary and are inevitably accompanied by noise interference. This makes it difficult to extract the feature frequency for the failed bearing and affects the diagnosis accuracy. The majorization-minimization-based total variation (TV-MM) denoising algorithm effectively removes the noise interference from the signal and highlights the related feature information. The value of its main parameter $\lambda$ determines the quality of the denoising effect. However, manually selecting parameters requires professional experience in a process that it is time-consuming and laborious, while the use of genetic algorithms is cumbersome. Therefore, an improved particle swarm algorithm (IPSO) is used to find the optimal solution of $\lambda$. The IPSO utilises the mutation concept in genetic algorithms to reinitialise the particles with a certain probability after each update. In addition, the empirical wavelet transform (EWT) is an adaptive signal processing method suitable for processing nonlinear and nonstationary signals. Therefore, this paper presents an ensemble analysis method that combines the IPSO, TV-MM, and EWT. First, IPSO is used to optimise the denoising parameter $\lambda$. The TV-MM under this parameter effectively removes the background noise interference and improves the accuracy of the subsequent modal decomposition. Then, the EWT is used for the adaptive division to produce a set of sequences. Finally, Hilbert envelope demodulation is performed on each component to realise fault diagnosis. The results from simulations and signals received from defective bearings with outer race fault, inner race fault, and rolling element fault demonstrate the effectiveness of the proposed method for fault diagnosis of rolling bearings.
\end{abstract}

\section{Introduction}

Mechanical fault diagnosis includes condition detection, fault prediction [1, 2], and analytical diagnosis [3]. Rolling bearings, which are the central component of mechanical systems, are a high failure point. Once a failure occurs, there is a fatal breakdown with significant consequences. Thus, research on rolling bearings is of particular interest in fault diagnosis. The failure signal gives periodic impulses with nonlinear and nonstationary properties.

Empirical mode decomposition (EMD) [4] is a method that is especially suitable to analyse and process nonlinear and nonstationary signals while also having a good time- frequency focus. Therefore, this approach has received attention from several scholars $[4,5]$. However, EMD comes with some problems such as modal aliasing, a large number of required calculations, and the lack of a complete theoretical foundation. Thus, scholars have begun to introduce alternative methods [5-8]. Among them, the empirical wavelet transform (EWT) was proposed by Gilles [8], which combines the wavelet transform and empirical mode decomposition. It is an adaptive time-frequency analysis algorithm and suitable for processing nonstationary signals.

The EWT adaptively divides the Fourier spectrum and uses a set of wavelet filters to obtain a single component. Envelope demodulation is then performed to obtain the 
corresponding instantaneous amplitude and frequency. This approach overcomes problems such as modal aliasing and false modalities and has a complete theoretical basis. Therefore, EWT has been applied in the field of fault diagnosis. Zhang [9] proposed a weak feature enhancement method, which incorporates EWT and an improved adaptive bistable stochastic resonance (IABSR). Experimental cases prove the effectiveness and superiority of the proposed method. Xu [10] used EWT and wavelet thresholding to filter out ECG motion artefact. The consequents of quantitative research and analysis show that this methodology produces a better performance in terms of restoring the QRS complexes of the original ECG with a reduced distortion, which retains useful information from the ECG signals. For the diagnosis of compound faults in an industrial wind turbine gearbox, Wei [11] utilised the EWT to adaptively seek a weak fault feature frequency in the planetary stage as well as evident fault characteristics in the other ordinary stages. The products demonstrate that EWT can intelligently extract multiple fault features. However, in actual engineering environments, it is typical for mechanical equipment operation to be disturbed by strong ambient noise, which is mixed with other machine components. This obscures the impact characteristics of the early failure vibration signal of the bearing and reduces the accuracy of the signal processing. If the fault signal is processed directly using this methodology, the diagnosis will fail. Hence, it is imperative to perform noise reduction preprocessing and highlight the impact characteristics of the fault signal.

Several signal processing methods have been developed to extract the true signal from a noisy signal. For example, Wang [12] proposed an enhanced vibration signal denoising method based on the dual-tree complex wavelet transform and NeighCoeff shrinkage, which could effectively remove noise. Flandrin [13] applied the Huang data-driven technique of empirical mode decomposition to the versatile and broadband model of fractional Gaussian noise. The experimental spectral analysis and statistical characterisation of the obtained modes revealed an equivalent filter bank structure. Xiang [14] proposed a hybrid approach using a probabilistic principal component analysis and spectral kurtosis to detect rolling element bearing faults. The signal to noise ratio (SNR) of the probabilistic principal component analysis (PPCA) denoising model is improved by selecting two key parameters. Then, a bandpass filter for the denoising signal was designed using the rapid spectral kurtosis (SK) procedure. The results indicate that their proposed method effectively detected faults for the rolling element bearing.

Meng [15] combined the morphological filter with a translation invariant wavelet, which was taken as the prefilter process unit to reduce the narrowband impulses and random noise in the original signal. The results show that their approach is feasible and effective for fault detection in rolling bearings. $\mathrm{Hu}$ [16] proposed an improved morphological filter (MF) algorithm to denoise a signal and obtain the fault features from low SNR signals. McDonald [17] proposed a maximum correlated Kurtosis deconvolution method. The experimental results indicate that with their improved performance, deconvolution of separate fault periods is possible, which allows for concurrent fault detection. The majorization-minimization-based total variation denoising algorithm (TV-MM) $[18,19]$ is an excellent denoising processing methodology that can effectively remove background noise while retaining detailed characteristics. To ensure the signal is not distorted and retains the desired features, the TV-MM seeks a minimum cost function of the signal and uses the maximum value of the weighted kurtosis as an indicator. The parameter $\lambda$ controls the weight of the total variation term, which significantly influences the denoising effects of the signatures. Over the years, scholars have also conducted some research on parameter detection and identification [20-22].

In this paper, an ensemble analysis method is proposed here to perform fault diagnosis. First, an improved particle swarm algorithm (IPSO) is used to optimise the selected denoising parameter. Further, the results are used in the TVMM for denoising preprocessing. Then, the denoised signal is decomposed adaptively using EWT. Finally, a Hilbert transform is performed to envelope demodulate the frequency characteristics of the bearing fault. This hybrid methodology combines the following advantages: fast optimised property for IPSO, TV-MM's excellent noise reduction performance, and EWT's ability to handle nonstationary as well as nonlinear signals. Thus, the method has practical application significance. Moreover, the experimental results of bearing fault diagnosis for doubly fed wind turbines further verify the effectiveness of this method.

The paper is outlined as follows. In Section 2, the concepts for EWT, IPSO, and TV-MM are briefly reviewed. Section 3 introduces the ensemble method, and Section 4 provides numerical simulation results to prove the effectiveness of the ensemble analysis algorithm. In Section 5, the research of the rolling bearings for MPT1000 further proves the validity of the method. Section 6 concludes the work and discusses further analysis.

\section{Theoretical Background}

2.1. A Brief Review of the EWT. The empirical wavelet transform (EWT) [8] decomposes a signal into a set of intrinsic modal functions, and the sum of residuals is defined as

$$
s(t)=\sum_{i=1}^{N} s_{i}(t)+r_{n}(t) .
$$

This method solves problems of modal aliasing and false modalities while also having an adaptive and complete theoretical basis. The operating procedure for EWT is described as follows:

(1) Determine the Fourier spectrum's division interval $\Lambda$ for $[0, \pi]$. Each segment can be written as

$$
\Lambda_{n}=\left[\omega_{n-1}, \omega_{n}\right] n=1,2 \ldots N
$$

where $\omega_{n}$ represents the centre of the two adjacent maxima. 
(2) Construct empirical wavelet functions $\Phi_{n}(\omega)$ and empirical scale functions $\Psi_{n}(\omega)$ using equations (3) and (4) below, where $\beta(x)$ and $\gamma$ are shown in equations (5) and (6). Use equations (3), (4), (5), and (6) to process each segmentation interval and obtain a set of empirical modes.
(3) Perform the Hilbert transform on each mode and display its instantaneous frequency and its instantaneous amplitude.

$$
\begin{aligned}
& \widehat{\Psi}_{n}(\omega)= \begin{cases}1 & |\omega| \leq(1-\gamma) \omega_{n} \\
\cos \left[\frac{\pi}{2} \beta\left(\frac{1}{2 \gamma \omega_{n}}\left(|\omega|-(1-\gamma) \omega_{n}\right)\right)\right] & (1-\gamma) \omega_{n} \leq|\omega| \leq(1+\gamma) \omega_{n} \\
0 & \text { otherwise, }\end{cases} \\
& \widehat{\Phi}_{n}(\omega)= \begin{cases}1 & (1+\gamma) \omega_{n} \leq|\omega| \leq(1-\gamma) \omega_{n+1}, \\
\cos \left[\frac{\pi}{2} \beta\left(\frac{1}{2 \gamma \omega_{n+1}}\left(|\omega|-(1-\gamma) \omega_{n+1}\right)\right)\right] & (1-\gamma) \omega_{n+1} \leq|\omega| \leq(1+\gamma) \omega_{n+1}, \\
\sin \left[\frac{\pi}{2} \beta\left(\frac{1}{2 \gamma \omega_{n}}\left(|\omega|-(1-\gamma) \omega_{n}\right)\right)\right] & (1-\gamma) \omega_{n} \leq|\omega| \leq(1+\gamma) \omega_{n}, \\
0 & \text { otherwise, }\end{cases} \\
& \beta(x)=x^{4}\left(35-84 x+70 x^{2}-20 x^{2}\right), \\
& \gamma<\min _{n}\left(\frac{\omega_{n-1}-\omega_{n}}{\omega_{n+1}+\omega_{n}}\right) .
\end{aligned}
$$

2.2. A Brief Review of the TV-MM. The key aspect of the majorization-minimization-based total variation denoising algorithm (TV-MM) [18] is to convert the problem of solving the minimum cost function, $D(x)$, into finding the minimum value of the upper boundary function, $B(x)$. In principle, the selection of the upper boundary function should satisfy the following conditions as much as possible:

(1) $D(x) \geq B(x), \forall x$.

(2) At the point $x_{k}, D\left(x_{k}\right)=B\left(x_{k}\right)$.

(3) $B(x)$ is a convex function.

(4) To reduce the computational complexity, replace the upper boundary function with a second-order polynomial, $B_{1}(x)$. Calculate equation (8) to obtain the results.

In the process of denoising, the kurtosis is widely used in fault diagnosis, and the maximum kurtosis value is regarded as a significant indicator of denoising, whereas, simply utilising the kurtosis as a measure of the denoising index may cause excessive denoising consequences, and the details in the signature may be removed. Consequently, the correlation coefficient is suppled to maintain a certain similarity before and after denoising. In addition, it can compensate for the distortion of signal in excessive pursuit of the kurtosis. The weighted kurtosis index, as a consequence, is used to make the denoised signal keep a balance between fidelity constraint and denoising.

From equation (13), the weighted kurtosis [19] is the product of the kurtosis index and the $r$-th power of the correlation coefficient index. The range of $\lambda$ is set to $[0,1]$.

$$
\begin{aligned}
& D(x)=\arg \min \left\{\frac{1}{2}\|f-x\|_{2}^{2}+\lambda\left\|D_{1} x\right\|\right\}, \quad x \in R, \\
& B_{1}(x)=x_{k+1}=\arg \min \frac{1}{2}\|f-x\|_{2}^{2}+l \frac{1}{2} x^{T} D_{1}^{T} \Lambda_{k}^{-1} D_{1} x \\
& +l \frac{1}{2}\left\|D_{1} x_{k}\right\|_{1} \\
& D_{1}=\left[\begin{array}{cccc}
-1 & 1 & & \\
& -1 & 1 & \\
& & 0 & 0 \\
& & -1 & 1
\end{array}\right] \\
& \Lambda_{k}=\left[\begin{array}{llll}
x_{k}(1) & & & \\
& \left|x_{k}(2)\right| & & \\
& & O & \\
& & & \left|x_{k}(N)\right|
\end{array}\right]=\operatorname{diag}\left(\left|x_{k}\right|\right),
\end{aligned}
$$




$$
\begin{aligned}
K_{-} I & =\frac{n \sum_{i=1}^{n}\left(x_{i}-\bar{x}\right)^{4}}{\left(\sum_{i=1}^{n}\left(x_{i}-\bar{x}\right)^{2}\right)^{2}}, \\
C_{-} I & =\frac{\operatorname{cov}(x, f)}{\sigma_{x} \sigma_{f}}=\frac{E((x-\bar{x})(f-\bar{f}))}{\sigma_{x} \sigma_{f}}, \\
K C_{-} I & =K_{-} I \times\left|C_{-} I\right|^{r},
\end{aligned}
$$

where $r$ is a positive real number that can be adjusted based on the actual situation and is used to control the fidelity of the output signal.

2.3. A Brief Review of the IPSO. The particle swarm optimisation (PSO) algorithm [23] simulates bird swarm foraging. In the swarm model of an $N$-dimensional search space, the position of particle $m$ is given by $P_{m}=\left(P_{m 1}, P_{m 2}\right.$, $\left.P_{m 3}, \ldots, P_{M N}\right)$ with a corresponding velocity of $V_{m}=\left(V_{m 1}\right.$, $\left.V_{m 2}, V_{m 3}, \ldots, V_{M N}\right) . m=1,2, \ldots, M$ is the particle population size, and $k$ is the number of iterations. The iterative update formula for the speed and position is expressed as

$$
\begin{aligned}
& V_{m n}^{k+1}=w V_{m n}^{k}+c_{1} r_{1}\left(G_{m n}^{k}-P_{m n}^{k}\right)+c_{2} r_{2}\left(S_{m n}^{k}-P_{m n}^{k}\right) \\
& P_{m n}^{k+1}=P_{m n}^{k}+V_{m n}^{k+1},
\end{aligned}
$$

where $r_{1}$ and $r_{2}$ are random coefficients between [0,1], $c_{1}$ and $c_{2}$ are nonnegative acceleration factors, and $w$ is the inertia weight. $G_{m}=\left(G_{m 1}, G_{m 2}, G_{m 3}, \ldots, G_{M N}\right)$ gives the best previous position of particle $m$, which is defined by the individual extreme values Gbest from the previous $k$ iterations. $S_{m}=\left(S_{m 1}, S_{m 2}, S_{m 3}, \ldots, S_{M N}\right)$ indicates the optimal position among particle $m$ 's neighbourhood, which is defined from the Gbest of the previous $k$ iterations. The algorithm first initialises the particle swarm $P_{m}$ in the $N$-dimensional solution space. Updating the particle position $P_{m}$ allows the velocity $V_{m}$ and fitness function to identify the individual extreme values Gbest and group extreme values Sbest to optimise the particles.

To prevent particles from falling into local extremes when searching for targets, the improved particle swarm optimisation (IPSO) algorithm is introduced mutate some particles after each iterative update, i.e., some particles are randomly extracted and reinitialised. The mutation operator not only expands the search space that constantly shrinks due to the iterations and helps particles trapped in local extremes to break free of their constraints but also improves the accuracy of the search targets.

\section{Ensemble Method}

3.1. Steps of the Ensemble Method. As is well known, the IPSO is an outstanding optimisation method that can search for an optimal value quickly and efficiently. As a highperformance denoising method, the TV-MM can remove noise components while maintaining signal distortion. The EWT is a kind of adaptive signal time-frequency analysis method that can effectively identify the modes that exist in the signal and can successfully decompose them. Hence, an ensemble methodology combining their respective advantages is proposed, which is called the improved majorization-minimization-based total variation and empirical wavelet transform. The implementation of the ensemble fault diagnosis method includes the four following steps:

(1) Optimise the selection of the denoising parameter using IPSO

(2) Substitute the selected parameter into the TV-MM to reduce the errors caused by noise

(3) Use EWT to adaptively decompose the signal

(4) Perform Hilbert envelope demodulation

A processing flow diagram is shown in Figure 1.

3.2. Denoising Method Based on Improved MajorizationMinimization-Based Total Variation. The TV-MM denoising algorithm is for high-performance approach to remove noise from a signal. The decisive factor quantifying its effect is an appropriate choice of the parameter $\lambda$.

The PSO $[24,25]$ is a relatively simple evolutionary computing technique in intelligent optimisation algorithms that avoids complex operations and uses the speed-displacement model to find an optimal solution. However, the algorithm can prematurely converge, and its iterative efficiency is not high in later periods of the algorithm. The IPSO uses the idea of mutation in genetic algorithms to reinitialise particles with a certain probability after each update to overcome these problems. Therefore, using the IPSO to quickly select the denoising parameter $\lambda$ ensures that the noise impact characteristics are retained while also filtering the noise. The accuracy of subsequent signal analyses is improved using this approach.

Next, a test function is used to visualise the performance of the proposed algorithm. Figure 2 shows the 3D surface plot of the cross-in-tray function, which is defined as

$$
f(x)=-0.0001\left(\left|f_{1}(x) \times f_{2}(x)\right|+1\right)^{0.1},
$$

where $f_{1}(x)$ and $f_{2}(x)$ are written as

$$
\begin{aligned}
& f_{1}(x)=\sin \left(x_{1}\right) \sin \left(x_{2}\right) \\
& f_{2}(x)=\exp \left(\left|100-\frac{\sqrt{x_{1}^{2}+x_{2}^{2}}}{\pi}\right|\right) .
\end{aligned}
$$

Due to the symmetry of the cross-in-tray function, the global minima of $f=-2.06261$ occurs at the 4 locations of (1.3491,-1.3491), (1.3491, 1.3491), (-1.3491, 1.3491), and $(-1.3491,-1.3491)$.

The fitness as calculated by the IPSO is $f=-2.063$, as shown in Figure 3. After approximately 15 generations, the optimal individual fitness stabilises with corresponding solutions of $(1.3491,-1.3491),(1.3491,1.3491),(-1.3491$, $1.3491)$, and $(-1.3491,-1.3491)$. It is seen that IPSO has a fast calculation speed, rapid convergence, and high accuracy for 


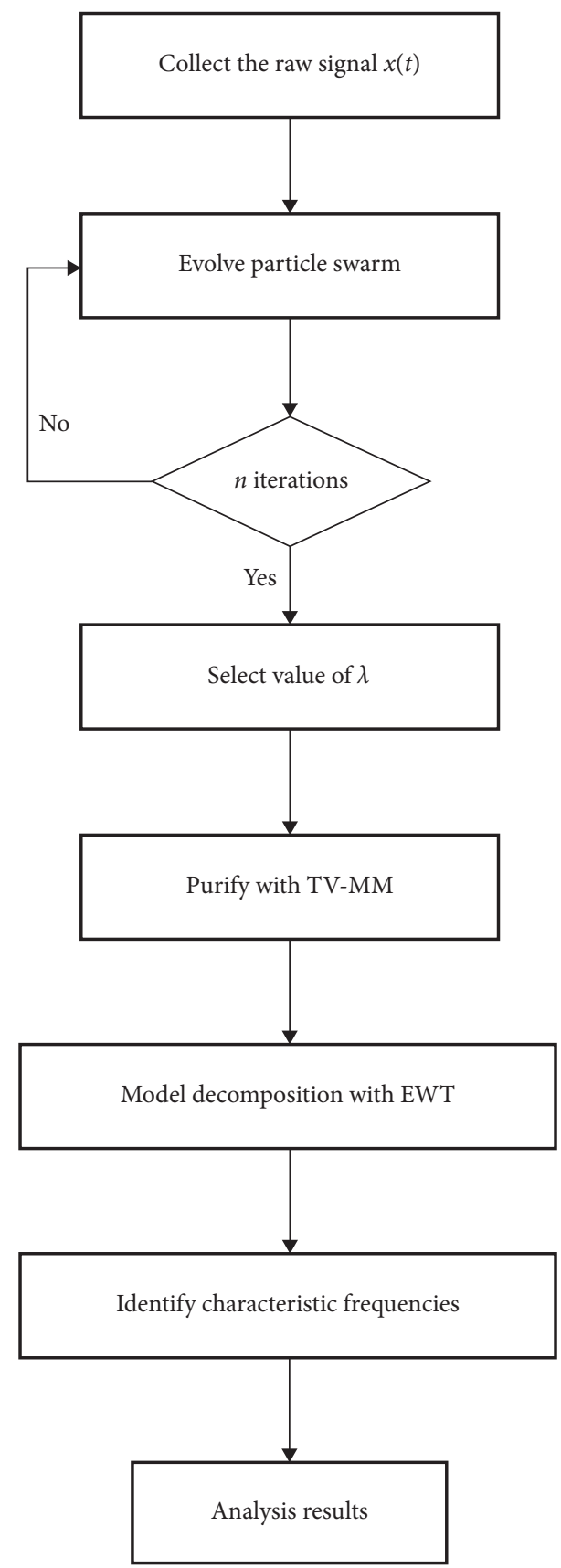

Figure 1: Processing flow diagram for the ensemble fault diagnosis method.

the calculated results. Thus, it can accurately obtain the optimal solution of the test function in a relatively short time. Hence, this approach is utilised to select the denoising parameter $\lambda$.

\section{Numerical Simulations}

A simulated signal with impact characteristics is constructed to verify the effectiveness of the proposed approach. The simulated signal is simplified as

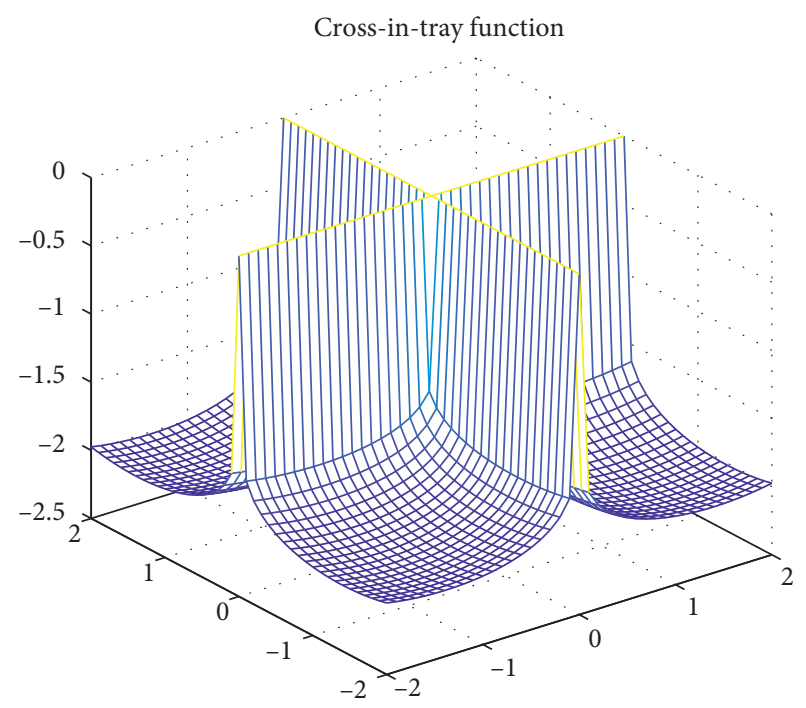

FIgURE 2: 3D surface plot of the cross-in-tray function.

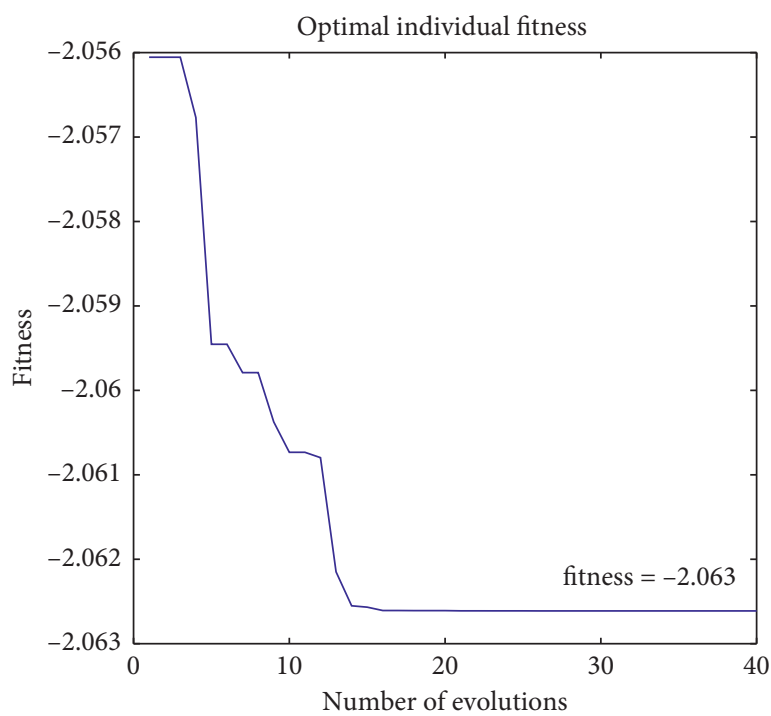

FIGURE 3: The search process for the optimal individual fitness.

$$
f(t)=f_{0} e^{-\zeta \omega_{n} t} \sin \left(\omega_{n} \sqrt{1-\xi^{2}} t\right)
$$

where the natural frequency is $f_{n}=3000 \mathrm{~Hz}$, the sampling frequency is $f_{s}=20 \mathrm{kHz}$, the number of sampling points is $N=2048, y_{0}=0.3$, the damping coefficient is $\zeta=0.1$, and the failure period is $0.01 \mathrm{~s}$. Considering the interference of the actual noise background environment, Gaussian noise is added to the simulated signal.

In the IPSO, the evolution number is 60 , the population size is 40 , and the mutation probability is 0.95 . After searching, the appropriate weighted kurtosis value is 0.074 , as shown in Figure 4, and the corresponding $\lambda$ is 0.25 . The wavelet threshold denoising approach [26-29] is also shown as processing the noisy signals as a comparison. The 4-level decomposition of the simulated signal uses the wavelet 


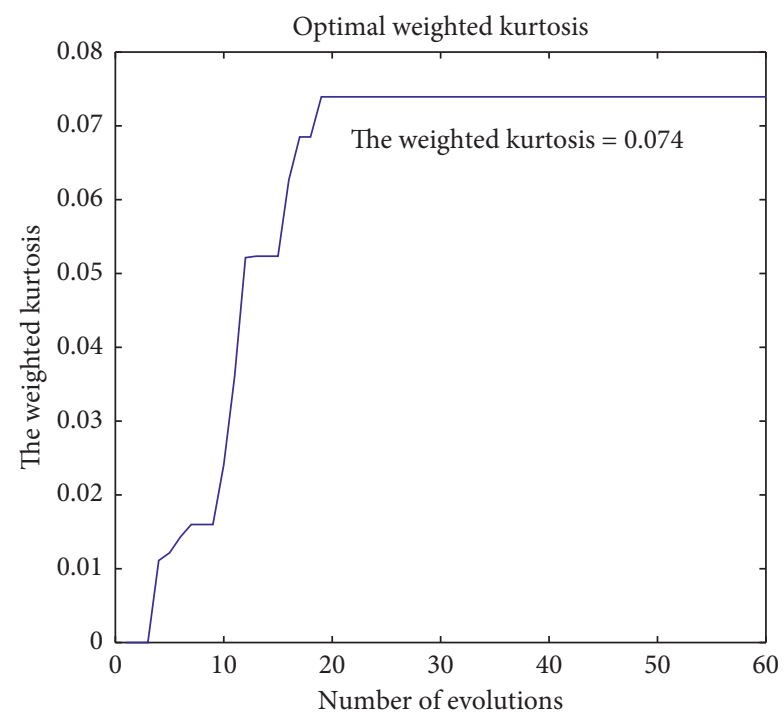

FIGURE 4: Search process for the optimal weighted kurtosis.

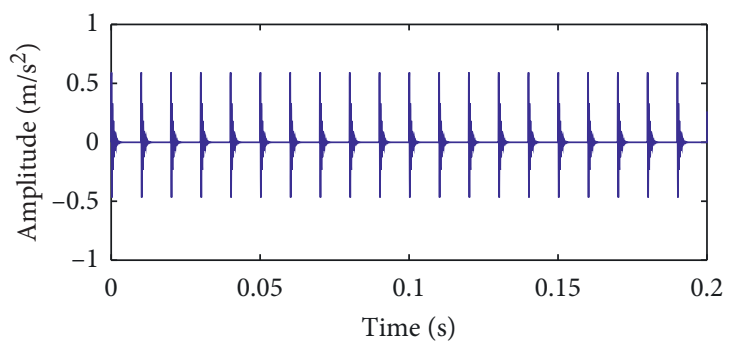

(a)

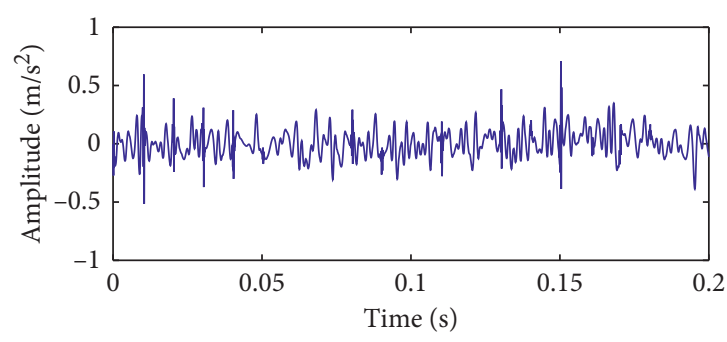

(c)

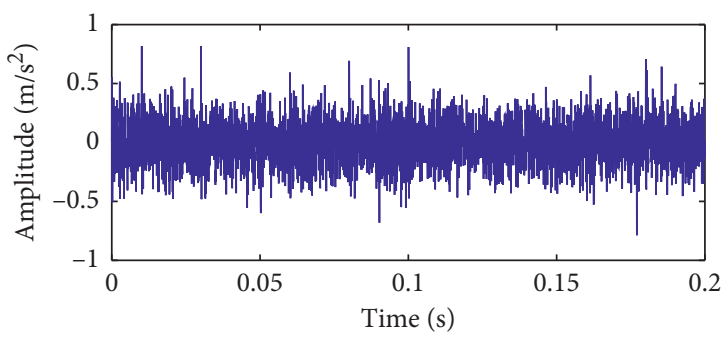

(b)

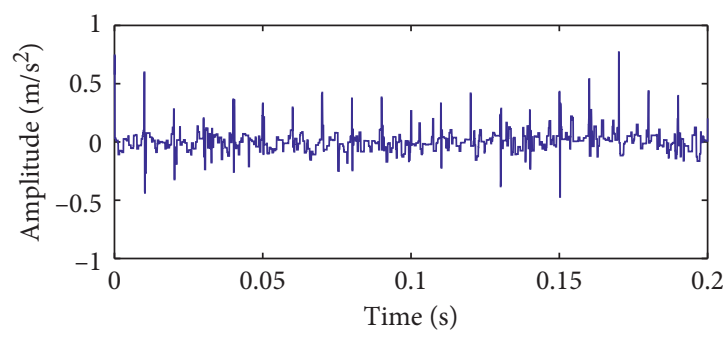

(d)

FIGURE 5: (a) The original signal, (b) signal with Gaussian noise, (c) the waveform of a purified signal with wavelet threshold denoising, and (d) the waveform of the purified signal using TV-MM.

function with $\mathrm{db} 3$ and a threshold of 0.323 . Figure 5(a) shows the pure simulated signal, and Figure 5(b) shows the simulated signal with added Gaussian noise. For the comparisons shown in Figure 5(c) and 5(d), the TV-MM is better than the wavelet denoising; the periodic impact signal after the TV-MM is more clear and the signal is not distorted. Hence, intelligent selection of the IPSO is effective and efficient.

The denoised signal is decomposed using the EMD and EWT. Compared with EMD, the EWT effectively decomposed the signals, and its modalities are more streamlined with neither false modes nor modal aliasing. However, each mode obtained via EMD has aliasing and false modes, as seen in Figure 6. Therefore, the decomposition mode performance with EWT is preferred over the EMD. The proposed ensemble methodology supplies a feasible plan for nonstationary signal analysis. The effectiveness of the proposed technique is illustrated with three practical examples given in the following section.

\section{Experimental Investigation}

A doubly fed wind turbine test platform (MPT1000) was used for experimentation to further validate the effectiveness of the proposed method. A photograph of the fault bearing component is shown in Figure 7. In the experiment, the 


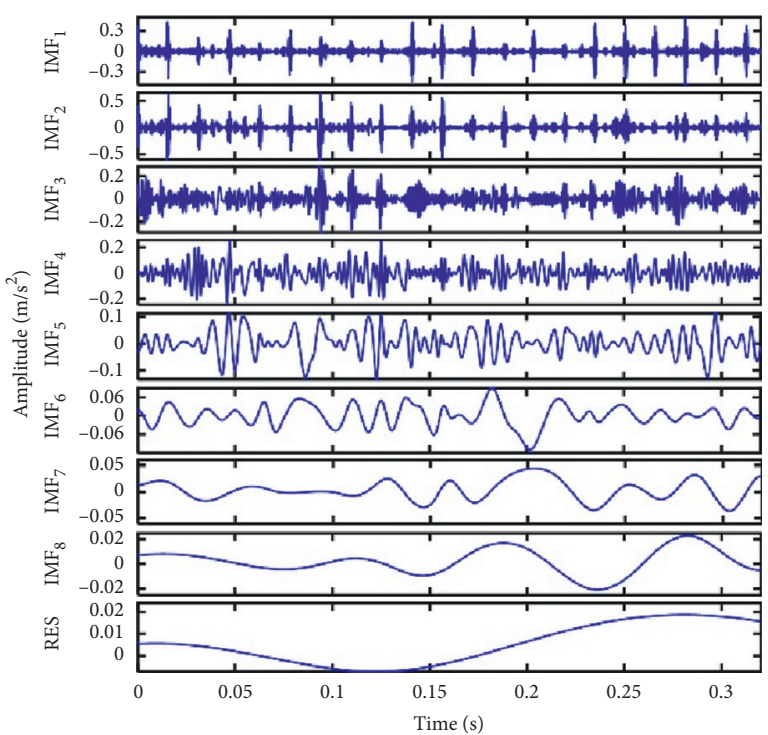

(a)

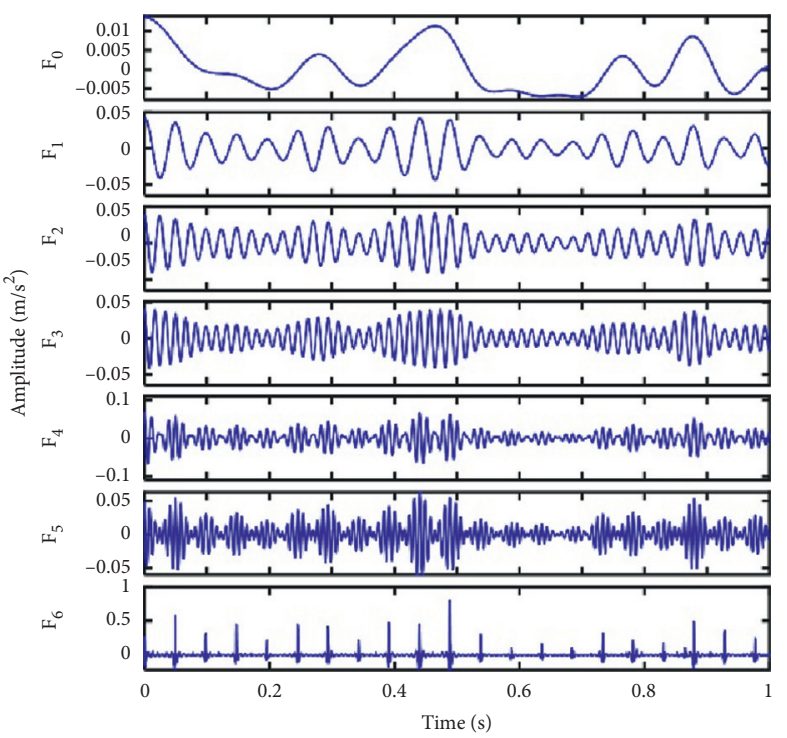

(b)

Figure 6: Modal components using (a) EMD and (b) EWT.

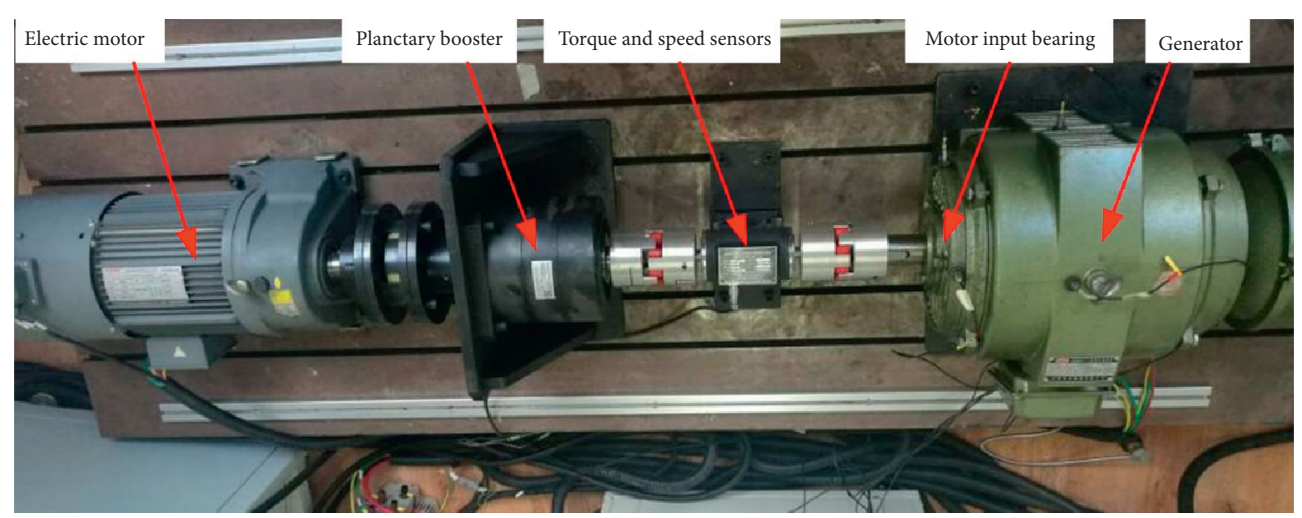

FIgURE 7: Test bench for testing the failed bearings.

NUP311E cylindrical roller bearing was used to detect the outer ring and rolling elements. The NJ311E cylindrical roller bearing was used to diagnose the inner ring. Table 1 provides the relevant bearing parameters.

The NUP311E cylindrical roller bearing has a $3 \mathrm{~mm}$ groove defection as processed with wire-cut machining technology to simulate a flaw in the outer race. One of the rolling elements of the NUP311E cylindrical roller bearing was selected for cutting to simulate rolling body failure. In addition, a $3 \mathrm{~mm}$ groove is set in the inner race of the NJ311E cylindrical roller bearing using the same processing technology. (a1), (b1), and (c1) of Figure 8 display glitches in the outer race, inner race, and rolling element, respectively. The formula to calculate the bearing theoretical fault characteristic frequency is shown in equations (20) to (22) [30]. The motor speed, sampling frequency, and bearing theoretical fault characteristic frequency are each described in Table 2. Due to assembly errors and other experiment factors, the actual value is not exactly equal to the theoretical value; thus, the theoretical calculation is only used as a diagnostic basis.

$$
\begin{aligned}
& f_{o}=\frac{1}{2} \times N_{b} \times f_{r} \times \pi\left(1-\frac{B_{d}}{P_{d}} \cos \theta\right) \\
& f_{i}=\frac{1}{2} \times N_{b} \times f_{r} \times \pi\left(1+\frac{B_{d}}{P_{d}} \cos \theta\right), \\
& f_{b}=\frac{1}{2} \times \frac{P_{d}}{B_{d}} \times f_{r} \times\left[1-\left(\frac{B_{d}}{P_{d}} \cos \theta\right)^{2}\right],
\end{aligned}
$$

where $N_{\mathrm{b}}$ is the number of rolling elements with diameter $B_{\mathrm{d}}$, $P_{\mathrm{d}}$ is the bearing pitch diameter, $\theta$ is the contact angle, and $f_{r}$ is the rotation frequency.

The time-domain waveform of the original outer race fault signal collected from the test platform is shown in (a2) 
TABLE 1: Parameters of NUP331E cylindrical roller rolling bearing.

\begin{tabular}{lcccccc}
\hline Model & Inside diameter $(\mathrm{mm})$ & Outer diameter $(\mathrm{mm})$ & $\begin{array}{c}\text { Rolling element } \\
\text { diameter }(\mathrm{mm})\end{array}$ & $\begin{array}{c}\text { The number } \\
\text { of rolling element }\end{array}$ & $\begin{array}{c}\text { The contact } \\
\text { angle }\left({ }^{\circ}\right)\end{array}$ & $\begin{array}{c}\text { Pitch } \\
\text { diameter }(\mathrm{mm})\end{array}$ \\
\hline NUP311E & 55 & 120 & 16.99 & 12 & 0 & 87.5 \\
NJ311E & 55 & 120 & 17 & 13 & 0 & 87.5 \\
\hline
\end{tabular}

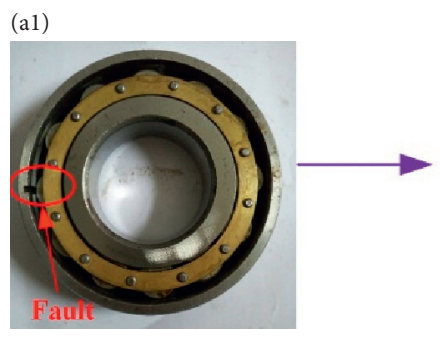

(b1)

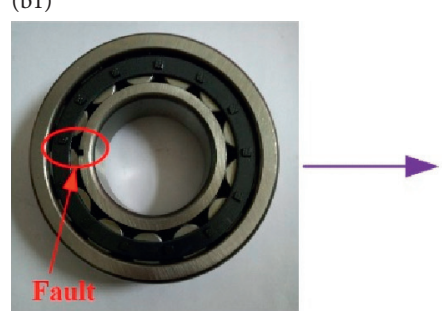

(c1)

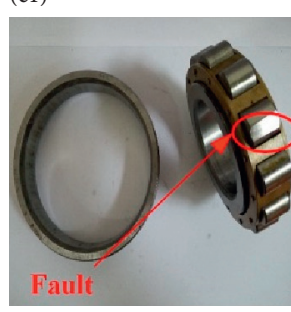

(a2)

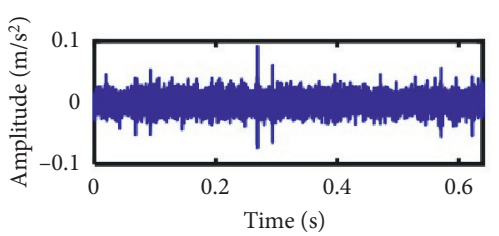

(a)

(b2)

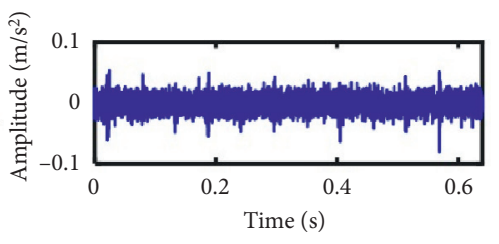

(b)

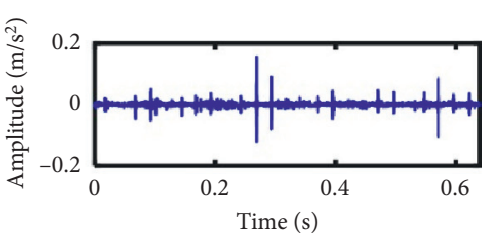

(b3)

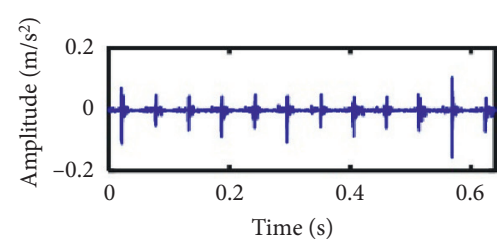

(c3)

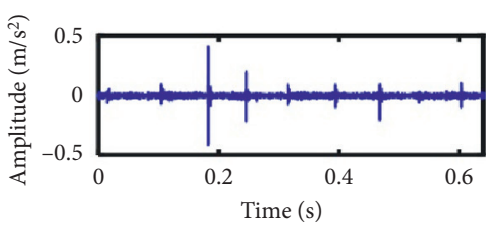

(c)

Figure 8: Fault bearings, signal collected from test bench, and their purified signals using TV-MM: (a) (a1) the outer race failure of NUP311E, (a2) measured outer race failure vibration signal, and (a3) purified signal using TV-MM; (b) (b1) inner race failure of NJ311E, (b2) measured inner race failure vibration signal, and (b3) purified signal using TV-MM; (c) (c1) rolling element failure of NUP311E, (c2) measured rolling element failure vibration signal, and (c3) purified signal using TV-MM.

TABLE 2: Experimental parameters of faulty bearings.

\begin{tabular}{lccccc}
\hline Fault model & $\begin{array}{c}\text { Motor speed } \\
(\mathrm{r} / \mathrm{min})\end{array}$ & $\begin{array}{c}\text { Motor output } \\
\text { frequency } \mathrm{f}_{\mathrm{op}}(\mathrm{Hz})\end{array}$ & Sampling frequency $(\mathrm{Hz})$ & Rotation frequency (Hz) & $\begin{array}{c}\text { Characteristic } \\
\text { frequency }(\mathrm{Hz})\end{array}$ \\
\hline Outer race failure & 1100 & 50 & 50 & 12800 & 18.3 \\
Inner race failure & 1100 & 50 & 12800 & 18.3 & 88.5 \\
Rolling element failure & 1100 & 12800 & 18.3 & 142 \\
\hline
\end{tabular}

of Figure 8(a). As is well known, the vibration signatures of faulted rolling bearings are usually saturated in random noise, and there is significant noise seen in (a2) of Figure 8(a). If the influence of noise is not filtered, the EWT is used to decompose the signal directly, which causes misjudgement and affects the accuracy of the subsequent fault diagnosis. Using the TV-MM first, which utilises the IPSO adaptive optimisation to select the denoising parameter $\lambda$, to eliminate the noise of the raw signal effectively suppresses the noise, as shown in (a3) of Figure 8(a). As a result, the impact characteristics of the signal can obviously be seen. The EWT decomposition and Hilbert envelope 


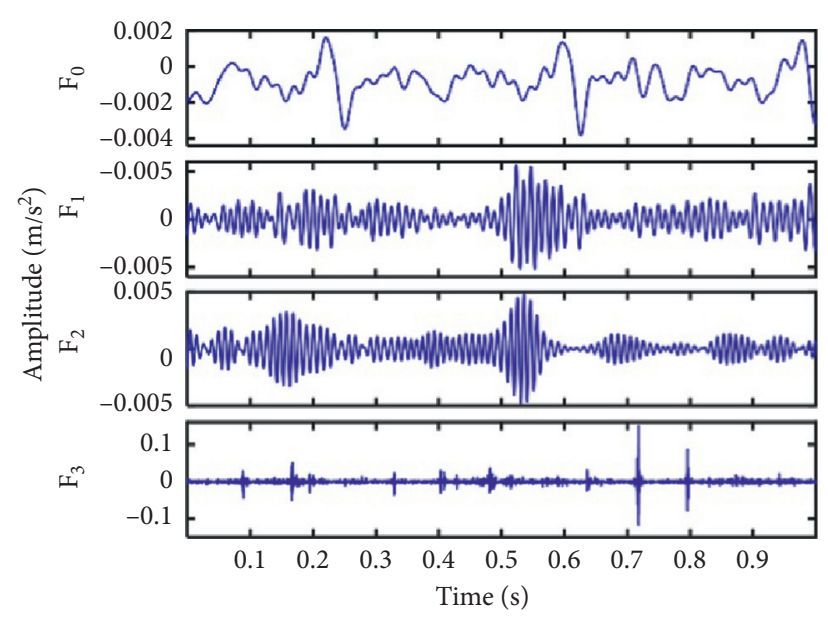

(a)

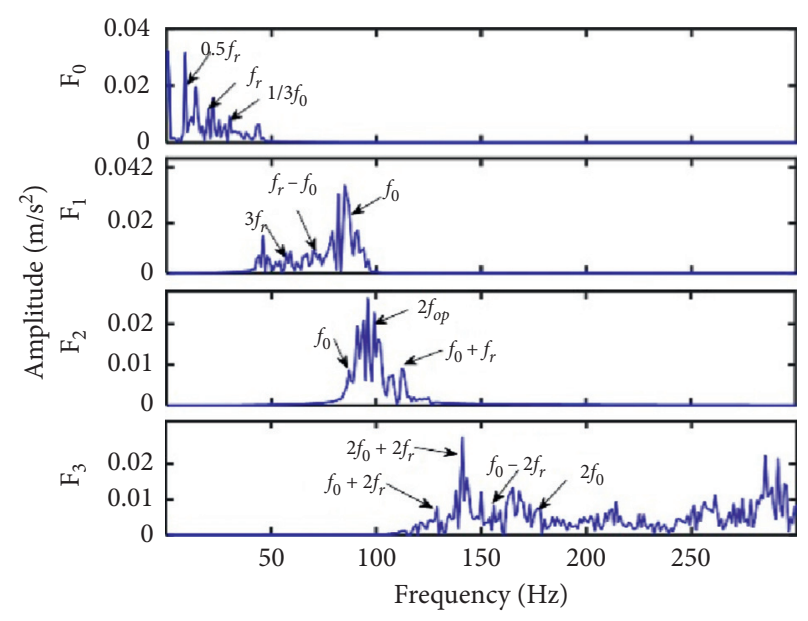

(b)

FIGURE 9: Outer race fault characteristics: (a) modal components of the outer race fault signal using EWT and (b) the spectrum of modal components.

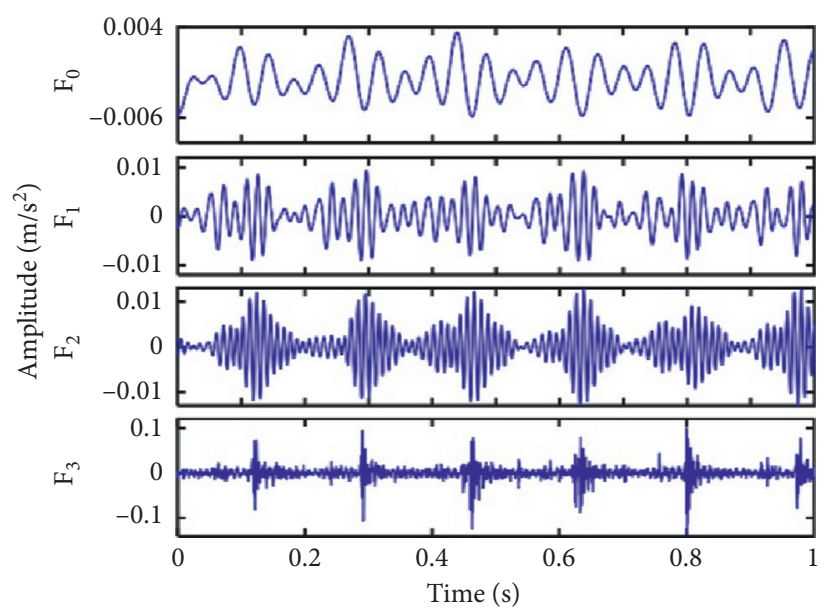

(a)

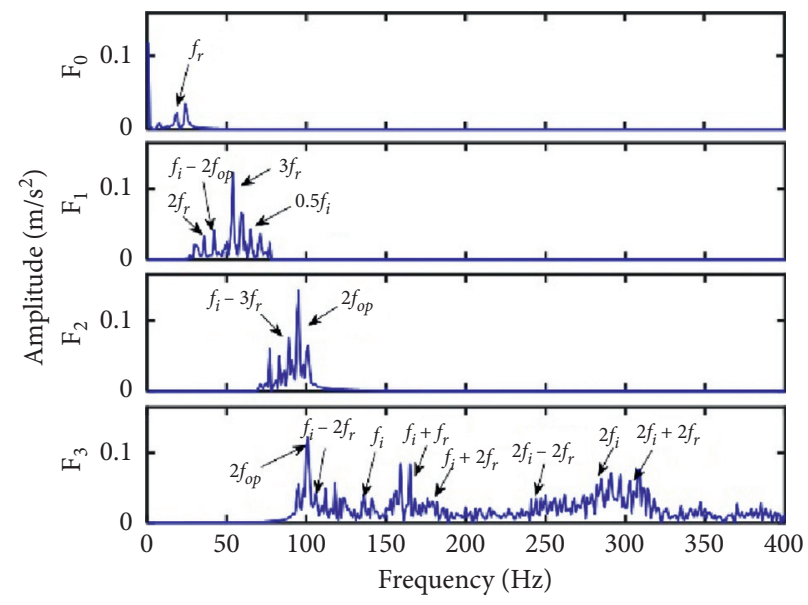

(b)

FIGURE 10: Inner race fault characteristics: (a) modal components of inner race fault signal using EWT and (b) their associated spectra.

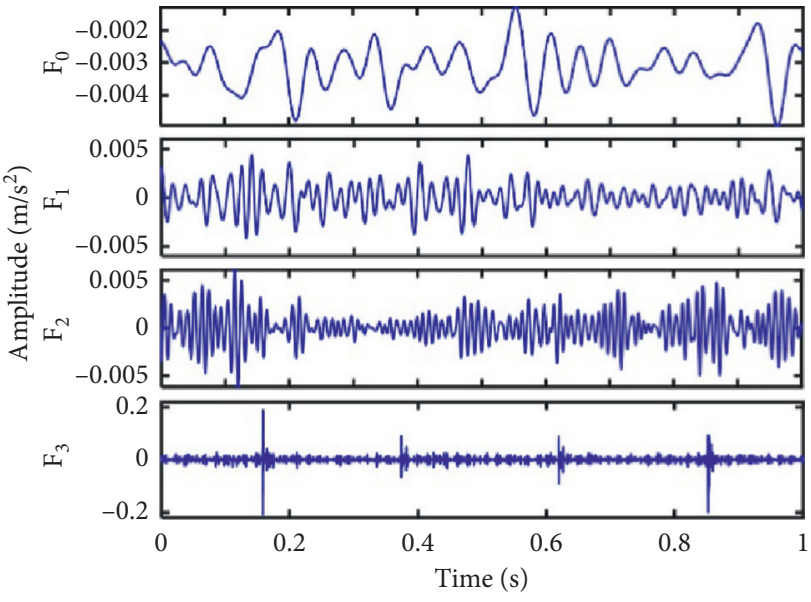

(a)

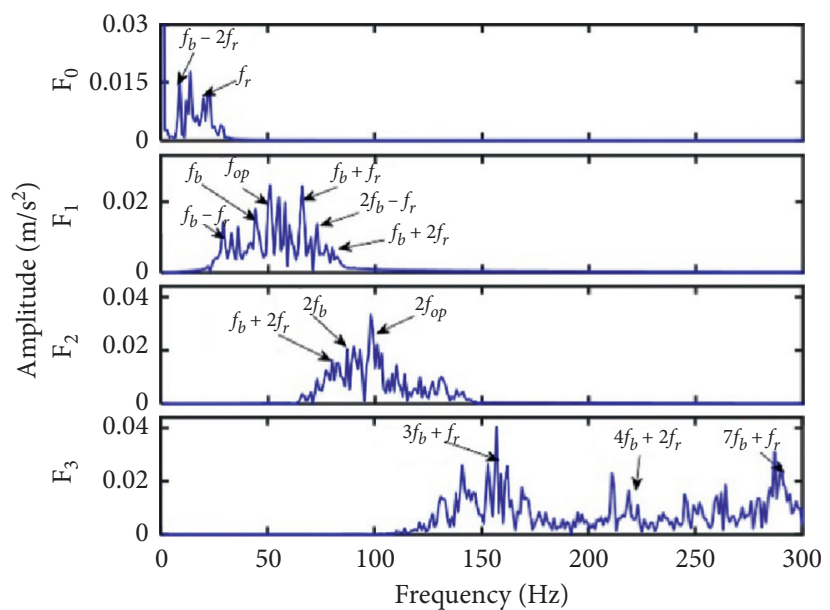

(b)

FIGURE 11: Rolling element fault characteristics: (a) modal components of the rolling element fault signal using EWT and (b) their associated spectra. 
analysis are performed to obtain the modal and frequency spectrum, as illustrated in Figures 9(a) and 9(b), respectively. The decomposition level is set to 4 , and the analysis data length is 4096 points. The modal components are clear and not mixed with some characteristic frequencies seen, i.e., $f_{r}$, $1 / 3 f_{o}, f_{o}-f_{r}, f_{o}, f_{o}+f_{r}, f_{o}+2 f_{r}, 2 f_{o}+2 f_{r}, f_{o}-2 f_{r}$, and $2 f_{o}$.

The background noise masks some of the useful information for the inner race fault signal measured from the test rig, as shown in (b2) of Figure 8(b). To derive the purified signal of the inner race vibration signature, the IPSO is exploited to select an optimal $\lambda$. The periodic impact signal becomes more obvious after the TV-MM denoising, as illustrated in (b3) of Figure 8(b). After the modal decomposition using the EWT, a set of frequencies are obtained in Figures $10(\mathrm{a})$ and $10(\mathrm{~b})$ as $f_{r}, f_{i}-2 f_{o p}, 0.5 f_{i}, f_{i}-3 f_{r}, f_{i}-2 f_{r}, f_{i}$, $f_{i}+f_{r}, f_{i}+2 f_{r}, 2 f_{i}$, and $2 f_{i}+f_{r}$.

As shown in (c2) and (c3) of Figure 8(c), for rolling element failure signals, the TV-MM uses the IPSO to select the parameter $\lambda$ for the denoising processing. This filters out the background noise and enhances the impact properties while preserving the details. Figures 11(a) and 11(b) show the spectra of the modal components and characteristic frequencies $f_{b}-2 f_{r}, f-f_{r}, f_{b}, f_{b}+f_{r}, 2 f_{b}-f_{r}, f_{b}+2 f_{r}, 2 f_{b}, 3 f_{b}+f_{r}$, etc. Thus, the above three experiments and the processing results of the proposed methodology prove the feasibility of bearing fault diagnosis.

\section{Conclusions}

Based on the superb properties of EWT, TV-MM, and IPSO, this paper proposes an ensemble analysis method for the fault detection of rolling bearings. The main advantages of this approach are that the IPSO can more efficiently obtain the denoising parameter, and after removing the noise interference with the TV-MM, the denoised signal still retains significant valuable information. Another advantage is that the computational efficiency of the EWT is greater than the EMD, and the EMD results show too many modalities and even exhibit modal aliasing. In addition, simulations were used to validate the TV-MM using the IPSO to derive the key parameter, which achieves a better performance than the wavelet denoising algorithm. In addition, the EWT is more powerful than EMD. The three types (outer race, inner race, and rolling element failure) of fault bearing signals were successfully detected with the proposed ensemble method. These confirm that the ensemble methodology is meaningful and suitable to detect faults in bearings. Moreover, enlarging $r$ by $n$ multiples of 10 can correct the phenomenon of excessive denoising.

Nonetheless, further work should consider finding the numerical relationship between $r$ and $\lambda$. In addition, the EWT can be further improved, such as through combinations with other methods to increase the accuracy of recognition.

\section{Data Availability}

The data used to support the findings of this study are available from the corresponding author upon request.

\section{Conflicts of Interest}

The authors declare that there are no conflicts of interest regarding the publication of this paper.

\section{Acknowledgments}

This research was financially supported by the Project of National Natural Science Foundation of China (grant no. 51965013), Science and Technology Major Project of Guangxi (grant nos. AA18242033 and AA18242036), Guangxi Key Laboratory of Manufacturing System and Advanced Manufacturing Technology (grant nos. 17-259-05-009Z and 17-259-05-010Z), Guangxi Science and Technology Base and Special Talents Program (grant no.2018AD19077), GUET Excellent Graduate Thesis (grant no. 17YJPYSS02), Guangxi Education Undergraduate Teaching Reform Project (2018JGA162), and Innovation Project of GUET Graduate Education (grant no. 2019YCXS001).

\section{References}

[1] S. Haidong, C. Junsheng, J. Hongkai, Y. Yu, and W. Zhantao, "Enhanced deep gated recurrent unit and complex wavelet packet energy moment entropy for early fault prognosis of bearing," Knowledge-Based Systems, vol. 188, pp. 105022105031, 2020.

[2] F. Chen, Y. Yang, B. Tang, B. Chen, W. Xiao, and X. Zhong, "Performance degradation prediction of mechanical equipment based on optimized multi-kernel relevant vector machine and fuzzy information granulation," Measurement, vol. 151, p. 107116, 2020.

[3] W. Song, J. Xiang, and Y. Zhong, "A simulation model based fault diagnosis method for bearings," Journal of Intelligent \& Fuzzy Systems, vol. 34, no. 6, pp. 3857-3867, 2018.

[4] N. E. Huang, "New method for nonlinear and nonstationary time series analysis: empirical mode decomposition and Hilbert spectral analysis," Proceedings of SPIE - The International Society for Optical Engineering, vol. 4056, pp. 197209, 2000.

[5] Y. Wang, Z. Wei, and J. Yang, "Feature trend extraction and adaptive density peaks search for intelligent fault diagnosis of machines," IEEE Transactions on Industrial Informatics, vol. 15, no. 1, pp. 105-115, 2019.

[6] Y. Wang, F. Liu, Z. Jiang, S. He, and Q. Mo, "Complex variational mode decomposition for signal processing applications," Mechanical Systems and Signal Processing, vol. 86, pp. 75-85, 2017.

[7] Y. Lei and M. J. Zuo, "Fault diagnosis of rotating machinery using an improved HHT based on EEMD and sensitive IMFs," Measurement Science and Technology, vol. 20, Article ID 125701, no. 12, pp. 1-12, 2009.

[8] J. GILLES, "Empirical wavelet transform," IEEE Transactions on Signal Processing, vol. 61, no. 16, pp. 3999-4010, 2013.

[9] X. Zhang, J. Wang, Z. Liu, and J. Wang, "Weak feature enhancement in machinery fault diagnosis using empirical wavelet transform and an improved adaptive bistable stochastic resonance," ISA Transactions, vol. 84, pp. 283-295, 2018.

[10] X. Xu, Y. Liang, P. He, and J. Yang, "Adaptive motion artifact reduction based on empirical wavelet transform and wavelet thresholding for the non-contact ECG monitoring systems," Sensors, vol. 19, no. 13, pp. 2916-2921, 2019. 
[11] W. Teng, X. Ding, H. Cheng, C. Han, Y. Liu, and H. Mu, "Compound faults diagnosis and analysis for a wind turbine gearbox via a novel vibration model and empirical wavelet transform," Renewable Energy, vol. 136, pp. 393-402, 2019.

[12] Y. Wang, Z. He, and Y. Zi, "Enhancement of signal denoising and multiple fault signatures detecting in rotating machinery using dual-tree complex wavelet transform," Mechanical Systems and Signal Processing, vol. 24, no. 1, pp. 119-137, 2010.

[13] P. Flandrin and P. Gonçalvès, "Empirical mode decompositions as data-driven wavelet-like expansions," International Journal of Wavelets, Multiresolution and Information Processing, vol. 2, no. 4, pp. 477-496, 2004.

[14] J. Xiang, Y. Zhong, and H. Gao, "Rolling element bearing fault detection using PPCA and spectral kurtosis," Measurement, vol. 75, pp. 180-191, 2015.

[15] L. Meng, J. Xiang, Y. Wang, Y. Jiang, and H. Gao, “A hybrid fault diagnosis method using morphological filter-translation invariant wavelet and improved ensemble empirical mode decomposition," Mechanical Systems and Signal Processing, vol. 50-51, pp. 101-115, 2015.

[16] Z. Hu, C. Wang, J. Zhu, X. Liu, and F. Kong, "Bearing fault diagnosis based on an improved morphological filter," Measurement, vol. 80, pp. 163-178, 2016.

[17] G. L. McDonald, Q. Zhao, and M. J. Zuo, "Maximum correlated Kurtosis deconvolution and application on gear tooth chip fault detection," Mechanical Systems and Signal Processing, vol. 33, pp. 237-255, 2012.

[18] M. A. T. Figueiredo, J. B. Dias, J. P. Oliveira et al., "On total variation denoising: a new majorization-minimization algorithm and an experimental comparison with wavelet denoising," in Proceedings of the International Conference On Image Processing, IEEE, Atlanta, GA, USA, pp. 2633-2636, October 2006.

[19] S. Zhang, Y. Wang, S. He, and Z. Jiang, "Bearing fault diagnosis based on variational mode decomposition and total variation denoising," Measurement Science and Technology, vol. 27, no. 7, pp. 1-10, 2016.

[20] W. Song, J. Xiang, Y. Zhong, and Y. Jiang, “A simulation based method to detect mechanical parameters of I-beams and hollow cylinders," Journal of Testing and Evaluation, vol. 47, pp. 1-20, 2020.

[21] W. Song, Y. Zhong, and J. Xiang, "Mechanical parameters identification for laminated composites based on the impulse excitation technique," Composite Structures, vol. 162, pp. 255-260, 2017.

[22] W. Song, J. Xiang, and Y. Zhong, "Mechanical parameters detection in stepped shafts using the FEM based IET," Smart Structures \& Systems, vol. 20, no. 4, pp. 473-481, 2017.

[23] J. Kennedy, R. Eberhart, and ", "Particle swarm optimization," Proceedings of the 1995 IEEE International Conference on Neural Networks, vol. 4, pp. 1942-1948, 1995.

[24] G. Garcia, G. Moreira, D. Menotti et al., "Inter-patient ECG heartbeat classification with temporal VCG optimized by PSO," Scientific Reports, vol. 7, no. 1, pp. 10543-10551, 2017.

[25] J. Wang and T. Kumbasar, "Parameter optimization of interval type-2 fuzzy neural networks based on PSO and BBBC methods," IEEE/CAA Journal of Automatica Sinica, vol. 6, no. 1, pp. 247-257, 2019.

[26] P. Chen and Q. Zhang, "Classification of heart sounds using discrete time-frequency energy feature based on S transform and the wavelet threshold denoising," Biomedical Signal Processing and Control, vol. 57, pp. 101684-101691, 2020.
[27] Q. Lu, L. Pang, H. Huang et al., "High-G calibration denoising method for high-G MEMS accelerometer based on EMD and wavelet threshold," Micromachines, vol. 10, no. 2, p. 134, 2019.

[28] J. Cai, "Gear fault diagnosis based on a new wavelet adaptive threshold de-noising method," Industrial Lubrication And Tribology, vol. 71, no. 1, pp. 40-47, 2019.

[29] O. El B'charri, R. Latif, and K. Elmansour, "ECG signal performance de-noising assessment based on threshold tuning of dual-tree wavelet transform," Biomedical Engineering Online, vol. 16, no. 1, p. 26, 2017.

[30] I. Bediaga, X. Mendizabal, A. Arnaiz, and J. Munoa, "Ball bearing damage detection using traditional signal processing algorithms," IEEE Instrumentation \& Measurement Magazine, vol. 16, no. 2, pp. 20-25, 2013. 